\title{
The Radial Wave Operator in Similarity Coordinates
}

\author{
Roland Donninger* \\ Faculty of Physics, Gravitational Physics, University of Vienna, Boltzmanngasse 5, A-1090 Wien
}

\begin{abstract}
We present a rigorous functional analytic setting to study the radial wave equation in similarity coordinates. As an application we analyse linear stability of the fundamental self-similar solution of the wave equation with a focusing power nonlinearity. This is part one in a series of papers, see Ref. 12, Ref. 13.
\end{abstract}

\section{INTRODUCTION}

\section{A. Motivation}

One of the most interesting properties of nonlinear wave equations is the development of singularities in finite time for solutions starting from smooth initial data. In a typical situation one observes that small initial data lead to dispersion whereas for large data the solution ceases to exist after a finite time by forming a singularity. An interesting mathematical question in this respect concerns the nature of the breakdown for generic large data solutions. It turns out that singularity formation is often connected to the existence of special solutions. In particular, for certain systems it has been observed that the breakdown occurs via a self-similar solution: Generic large data evolutions approach a certain self-similar solution and blow up. Such a knowledge is often based on numerical studies. We remark that the convergence to the special solution is due to dispersion and can in general only hold in a local sense since the total energy is conserved. Obviously, the stability of special solutions plays a crucial role for understanding the dynamics.

As a first step in the analysis of such a system one studies linear stability of the solution which is expected to act as an attractor. However, unlike in the case of static solutions, the linearization around a self-similar solution yields an equation which depends explicitly on time. An elegant way to overcome this difficulty is to introduce adapted coordinates which transform the problem of self-similar blow up into an asymptotic stability problem. Such similarity coordinates have been successfully used by various authors (e.g. Ref. 2, Ref. 8, Ref. 9, Ref. 11, Ref. 10, Ref. 5) to study self-similar blow up behaviour for the semilinear wave equation.

The main motivation of the present paper is to obtain a systematic functional analytic initial value formulation of the wave equation in similarity coordinates. For simplicity we restrict ourselves to the radial case. The main difficulty one encounters is the fact that the spatial part of the wave operator in similarity coordinates is not normal. Therefore, standard spectral theory is not applicable. However, we are still able to obtain a well-posed initial value formulation by means of semigroup theory. This approach leads to interesting non self-adjoint spectral problems. In particular, we show that the generator of the semigroup possesses a continuum of eigenvalues filling a complex left half plane. As an application we consider the wave equation with a power nonlinearity where we study the linearization around the fundamental self-similar solution and show that it is linearly stable. This is achieved by a detailed study of the spectrum of the associated linear operator. This operator has exactly one unstable eigenvalue which is shown to emerge from the time translation symmetry of the orginal problem. We get rid of this instability by defining an appropriate projection that removes the unstable eigenvalue from the spectrum. Then we show that the resulting operator generates a semigroup and by analysing its resolvent we deduce appropriate growth bounds to conclude linear stability of the fundamental self-similar solution. The framework developed in the present work is fundamental for the follow-up papers Ref. 12 and Ref. 13 where the full nonlinear stability of the fundamental self-similar solution is proved.

\section{B. Notations}

Throughout this paper we will make use of the following notations. For a Hilbert space $H$ we denote by $\mathcal{B}(H)$ the Banach space of linear bounded operators on $H$. For a closed linear operator $L: \mathcal{D}(L) \subset H \rightarrow H$ we set $R_{L}(\lambda):=(\lambda-L)^{-1}$ if $(\lambda-L)$ is bijective. The spectrum $\sigma(L)$ of $L$ is decomposed as $\sigma(L)=\sigma_{p}(L) \cup \sigma_{c}(L) \cup \sigma_{r}(L)$ where

*Electronic address: roland.donninger@univie.ac.at 
- $\sigma_{p}(L)$ is the set of all eigenvalues of $L$,

- $\lambda \in \sigma_{c}(L)$ if $\lambda-L$ is injective but not surjective and the range of $\lambda-L$ is dense in $H$,

- $\lambda \in \sigma_{r}(L)$ if $\lambda-L$ is injective and the range of $\lambda-L$ is not dense in $H$.

The resolvent set of $L$ is denoted by $\rho(L)$. In order to improve readability, we write vectors as bold face letters and the individual components are numbered using lower indices, e.g. $\mathbf{u}=\left(u_{1}, u_{2}\right)^{T}$. Finally, the letter $C$ stands for a positive constant, however, this constant is not supposed to have the same value at each occurence.

\section{THE FREE WAVE EQUATION}

\section{A. Similarity Coordinates}

Consider the free wave equation in spherical symmetry, i.e.

$$
\tilde{\psi}_{t t}-\tilde{\psi}_{r r}-\frac{2}{r} \tilde{\psi}_{r}=0
$$

together with initial data $\tilde{\psi}(0, \cdot), \tilde{\psi}_{t}(0, \cdot)$. The simple transformation $\tilde{\psi} \mapsto \psi$ where $\psi(t, r):=r \tilde{\psi}(t, r)$ yields the one-dimensional wave equation $\psi_{t t}-\psi_{r r}=0$ together with the regularity condition $\psi(t, 0)=0$ for all $t$. Our aim is to study this equation in the coordinate system $(\tau, \rho)$, defined by $\tau:=-\log (T-t)$ and $\rho:=\frac{r}{T-t}$, which is adapted to self-similarity and we restrict ourselves to the backward lightcone of the point $(T, 0)$ for a $T>0$. Note that the transformation $(t, r) \mapsto(\tau, \rho)$ maps the cone $\{(t, r): 0 \leq t \leq T, 0 \leq r \leq T-t\}$ to the cylinder $\{(\tau, \rho): \tau \geq-\log T, \rho \in[0,1]\}$ and thus, $\tau \rightarrow \infty$ corresponds to $t \rightarrow T-$. The inverse transformation is given by $t=T-e^{-\bar{\tau}}$ and $r=\rho e^{-\tau}$. Furthermore, we note that the derivatives are given by $\partial_{t}=e^{\tau}\left(\partial_{\tau}+\rho \partial_{\rho}\right)$ and $\partial_{r}=e^{\tau} \partial_{\rho}$. We introduce the new dependent variables $\psi_{1}:=\psi_{t}$ and $\psi_{2}:=\psi_{r}$. Then, $\Psi:=\left(\psi_{1}, \psi_{2}\right)^{T}$ satisfies

$$
\partial_{t} \Psi=\left(\begin{array}{cc}
0 & 1 \\
1 & 0
\end{array}\right) \partial_{r} \Psi
$$

with the regularity condition $\psi_{1}(t, 0)=0$ for all $t$. In similarity coordinates $(\tau, \rho)$ this system reads

$$
\partial_{\tau} \Phi=\left(\begin{array}{cc}
-\rho & 1 \\
1 & -\rho
\end{array}\right) \partial_{\rho} \Phi
$$

where $\Phi(\tau, \rho)=\Psi\left(T-e^{-\tau}, \rho e^{-\tau}\right)$. The field $\phi(\tau, \rho):=\psi\left(T-e^{-\tau}, \rho e^{-\tau}\right)$ can be reconstructed from $\phi_{2}$ by noting that $\phi_{\rho}(\tau, \rho)=e^{-\tau} \phi_{2}(\tau, \rho)$ which yields

$$
\phi(\tau, \rho)=e^{-\tau} \int_{0}^{\rho} \phi_{2}(\tau, \xi) d \xi
$$

Now suppose we are able to deduce a growth bound for $\Phi$ of the form $\|\Phi(\tau, \cdot)\| \leq C e^{\mu \tau}$ for a $\mu \in \mathbb{R}$ where $\|\cdot\|$ is the norm on $L^{2}(0,1)^{2}$. What does this imply for the original system? Consider the energy of the original field $\tilde{\psi}$ in the backward lightcone of the point $(T, 0)$ which is given by

$$
\int_{0}^{T-t}\left[\psi_{t}^{2}(t, r)+\left(\psi_{r}(t, r)-\frac{\psi(t, r)}{r}\right)^{2}\right] d r
$$

in terms of $\psi$. Switching to similarity coordinates $(\tau, \rho)$ this expression reads

$$
E_{\phi}(\tau):=e^{-\tau} \int_{0}^{1}\left[\phi_{1}^{2}(\tau, \rho)+\left(\phi_{2}(\tau, \rho)-\frac{e^{\tau}}{\rho} \phi(\tau, \rho)\right)^{2}\right] d \rho .
$$

Applying the Hardy inequality to Eq. (2) we conclude that

$$
\int_{0}^{1}\left|\frac{\phi(\tau, \rho)}{\rho}\right|^{2} d \rho \leq C e^{-2 \tau}\left\|\phi_{2}(\tau, \cdot)\right\|_{L^{2}(0,1)}^{2}
$$


and the growth bound $\|\Phi(\tau, \cdot)\| \leq C e^{\mu \tau}$ implies $E_{\phi}(\tau) \leq C e^{2\left(\mu-\frac{1}{2}\right) \tau}$. Thus, if $\mu \leq \frac{1}{2}, E_{\phi}(\tau)$ stays bounded for all $\tau$ and the zero solution is stable in the energy space. Of course, for the free wave equation this stability is trivial, however, for our purposes it is useful to "reprove" it by deducing an appropriate growth bound for $\Phi$ which we are going to do in the following.

Furthermore, from $\left\|\phi_{2}(\tau, \cdot)\right\|_{L^{2}(0,1)} \leq C e^{\mu \tau}$ and Eq. (2) we conclude

$$
|\phi(\tau, \rho)| \leq e^{-\tau}\left\|\phi_{2}(\tau, \cdot)\right\|_{L^{2}(0,1)} \leq C e^{(\mu-1) \tau}
$$

by Cauchy-Schwarz and thus, $\|\phi(\tau, \cdot)\|_{L^{\infty}(0,1)} \leq C e^{(\mu-1) \tau}$. Therefore, the zero solution is asymptotically stable in the $L^{\infty}$ norm if $\mu<1$. In the original coordinates $(t, r)$ this estimate translates into $\|\psi(t, \cdot)\|_{L^{\infty}(0, T-t)} \leq C(T-t)^{1-\mu}$.

\section{B. Operator Formulation and Generation of Semigroup}

In order to deduce growth bounds for $\Phi$ we apply semigroup theory, i.e. we derive an operator formulation for Eq. (11). Let $\mathcal{H}:=L^{2}(0,1)^{2}$ and define $\mathcal{D}\left(\tilde{L}_{0}\right):=\left\{\mathbf{u} \in C^{1}[0,1]^{2}: u_{1}(0)=0\right\}, \tilde{L}_{0} \mathbf{u}:=\left(-\rho u_{1}^{\prime}+u_{2}^{\prime}, u_{1}^{\prime}-\rho u_{2}^{\prime}\right)^{T}$. Then $\tilde{L}_{0}: \mathcal{D}\left(\tilde{L}_{0}\right) \subset \mathcal{H} \rightarrow \mathcal{H}$ is a densely defined linear operator. The inner product and the norm on $\mathcal{H}$ are denoted by $(\cdot \mid \cdot)$ and $\|\cdot\|$, respectively. Thus,

$$
\frac{d}{d \tau} \Phi(\tau)=\tilde{L}_{0} \Phi(\tau)
$$

for a function $\Phi:\left[\tau_{0}, \infty\right) \rightarrow \mathcal{H}$ is an operator formulation of Eq. (11) where $\tau_{0}:=-\log T$, i.e. $\tau=\tau_{0}$ corresponds to $t=0$. In what follows we prove that the closure of $\tilde{L}_{0}$ generates a strongly continuous one-parameter semigroup $S_{0}:[0, \infty) \rightarrow \mathcal{B}(\mathcal{H})$ and thus, the unique solution of Eq. (3) is given by $\Phi\left(\tau+\tau_{0}\right)=S_{0}(\tau) \Phi\left(\tau_{0}\right)$.

Lemma 1. The operator $\tilde{L}_{0}$ satisfies $\operatorname{Re}\left(\tilde{L}_{0} \mathbf{u} \mid \mathbf{u}\right) \leq \frac{1}{2}\|\mathbf{u}\|^{2}$ for all $\mathbf{u} \in \mathcal{D}\left(\tilde{L}_{0}\right)$.

Proof. Integration by parts yields

$$
\begin{array}{r}
\operatorname{Re}\left(\tilde{L}_{0} \mathbf{u} \mid \mathbf{u}\right)=-\sum_{j=1}^{2} \operatorname{Re} \int_{0}^{1} \rho u_{j}^{\prime}(\rho) \overline{u_{j}(\rho)} d \rho+\operatorname{Re} \int_{0}^{1}\left(u_{2}^{\prime}(\rho) \overline{u_{1}(\rho)}+u_{1}^{\prime}(\rho) \overline{u_{2}(\rho)}\right) d \rho \\
=\frac{1}{2}\|\mathbf{u}\|^{2}-\frac{1}{2}\left|u_{1}(1)\right|^{2}-\frac{1}{2}\left|u_{2}(1)\right|^{2}+\operatorname{Re} \overline{u_{1}(1)} u_{2}(1) \\
+\operatorname{Re}\left(2 i \operatorname{Im} \int_{0}^{1} u_{1}^{\prime}(\rho) \overline{u_{2}(\rho)} d \rho\right) \leq \frac{1}{2}\|\mathbf{u}\|^{2} .
\end{array}
$$

Lemma 2. The range of $1-\tilde{L}_{0}$ is dense in $\mathcal{H}$.

Proof. Let $\mathbf{f} \in C^{\infty}[0,1]^{2}$ and define $\mathbf{u}$ by

$$
u_{2}(\rho):=\frac{1}{1-\rho^{2}} \int_{\rho}^{1} F(\xi) d \xi
$$

and $u_{1}(\rho):=\rho u_{2}(\rho)-\int_{0}^{\rho} f_{2}(\xi) d \xi$ where $F(\rho):=f_{1}(\rho)+\rho f_{2}(\rho)+\int_{0}^{\rho} f_{2}(\xi) d \xi$. An application of de l'Hospital's rule shows that $u_{2} \in C^{1}[0,1]$ and obviously, $u_{1} \in C^{1}[0,1], u_{1}(0)=0$. Hence, $\mathbf{u} \in \mathcal{D}\left(\tilde{L}_{0}\right)$ and a direct computation shows $\left(1-\tilde{L}_{0}\right) \mathbf{u}=\mathbf{f}$. Thus, the claim follows from the density of $C^{\infty}[0,1]^{2}$ in $\mathcal{H}$.

Proposition 1. The operator $\tilde{L}_{0}$ is closable and its closure $L_{0}$ generates a strongly continuous semigroup $S_{0}:[0, \infty) \rightarrow$ $\mathcal{B}(\mathcal{H})$ satisfying $\left\|S_{0}(\tau)\right\| \leq e^{\frac{1}{2} \tau}$.

Proof. The claim follows from Lemmas 1, 2 and the Lumer-Phillips Theorem (see e.g. Ref. 1, p. 56, Theorem 4.2.6).

Hence, this semigroup description yields the appropriate functional analytic setting to study the wave equation in similarity coordinates. According to the discussion in Sec. IIA, the growth bound $\left\|S_{0}(\tau)\right\| \leq e^{\frac{1}{2} \tau}$ implies the wellknown stability of the zero solution in energy space. Furthermore, we conclude that the zero solution is asymptotically stable with respect to the $L^{\infty}$ norm. 


\section{Spectral Analysis of the Generator}

We study the spectrum of $L_{0}$. To this end we need a more explicit description of $\mathcal{D}\left(L_{0}\right)$. We define the formal matrix differential expression $\ell_{0} \mathbf{u}:=\left(-\rho u_{1}^{\prime}+u_{2}^{\prime}, u_{1}^{\prime}-\rho u_{2}^{\prime}\right)^{T}$, i.e. $\ell_{0}$ generates $L_{0}$.

Lemma 3. The domain of $L_{0}$ is given by

$$
\mathcal{D}\left(L_{0}\right)=\left\{\mathbf{u} \in \mathcal{H}: \mathbf{u} \in H_{\mathrm{loc}}^{1}(0,1)^{2}, \ell_{0} \mathbf{u} \in \mathcal{H}, u_{1}(0)=0\right\} .
$$

Proof. Suppose $\mathbf{u} \in \mathcal{D}\left(L_{0}\right)$. Then there exists a sequence $\left(\mathbf{u}_{j}\right) \subset \mathcal{D}\left(\tilde{L}_{0}\right)$ such that $\mathbf{u}_{j} \rightarrow \mathbf{u}$ and $\tilde{L}_{0} \mathbf{u}_{j} \rightarrow L_{0} \mathbf{u}$ in $\mathcal{H}$. This implies that $\left(\left(1-\rho^{2}\right) u_{1 j}^{\prime}\right)$ and $\left(\left(1-\rho^{2}\right) u_{2 j}^{\prime}\right)$ are Cauchy sequences in $L^{2}(0,1)$. Thus, $u_{1}, u_{2} \in H^{1}(0,1-\delta)$ for any $\delta \in(0,1)$ and the Sobolev embedding $H^{1}(0,1-\delta) \hookrightarrow C[0,1-\delta]$ yields $u_{1}(0)=0$.

Conversely, if $\mathbf{u} \in \mathcal{H} \cap H_{\mathrm{loc}}^{1}(0,1)^{2}, \ell_{0} \mathbf{u} \in \mathcal{H}$ and $u_{1}(0)=0$ then $\left(1-\ell_{0}\right) \mathbf{u} \in \mathcal{H}$ and we define $\mathbf{v}:=R_{L_{0}}(1)\left(1-\ell_{0}\right) \mathbf{u}$. Then $\mathbf{v} \in \mathcal{D}\left(L_{0}\right)$ and by definition we have $\left(1-\ell_{0}\right) \mathbf{v}=\left(1-\ell_{0}\right) \mathbf{u}$. From the first part of the proof we conclude that $v_{1}(0)=0$ and a short calculation shows that $\left(1-\ell_{0}\right)(\mathbf{u}-\mathbf{v})=0$ necessarily implies $\mathbf{u}=\mathbf{v} \in \mathcal{D}\left(L_{0}\right)$.

Next we show that the analysis of the spectrum of $L_{0}$ can be reduced to the study of the invertibility of an operator-valued function. For $\lambda \in \mathbb{C}$ we define the formal differential expression $t_{0}(\lambda)$ by

$$
t_{0}(\lambda) u(\rho):=-\left(1-\rho^{2}\right) u^{\prime \prime}(\rho)+2 \lambda \rho u^{\prime}(\rho)+\lambda(\lambda-1) u(\rho)
$$

and set $\mathcal{D}\left(T_{0}(\lambda)\right):=\left\{u \in H^{1}(0,1): u \in H_{\text {loc }}^{2}(0,1), t_{0}(\lambda) u \in L^{2}(0,1), u(0)=0\right\}, T_{0}(\lambda) u:=t_{0}(\lambda) u$. The following result shows that $\sigma\left(L_{0}\right)$ is completely determined by $T_{0}(\lambda)$.

Proposition 2. The operator $\lambda-L_{0}$ for a $\lambda \in \mathbb{C}$ is bounded invertible if and only if $T_{0}(\lambda)$ is invertible. Furthermore, $\lambda \in \sigma_{p}\left(L_{0}\right)$ if and only if $\operatorname{dim} \operatorname{ker} T_{0}(\lambda)=1$. In this case, $\mathbf{u}=\left(u_{1}, u_{2}\right)^{T}$ defined by $u_{1}(\rho):=\rho u^{\prime}(\rho)+(\lambda-1) u(\rho)$ and $u_{2}:=u^{\prime}$ for $u \in \operatorname{ker} T_{0}(\lambda), u \neq 0$, is an eigenfunction of $L_{0}$.

Proof. Suppose $\lambda \in \sigma_{p}\left(L_{0}\right)$ and $\mathbf{u}$ is the associated eigenfunction. Then, $u_{1}^{\prime}=\lambda u_{2}+\rho u_{2}^{\prime}$ and this implies $u_{1}(\rho)=$ $\rho u_{2}(\rho)+(\lambda-1) \int_{0}^{\rho} u_{2}(\xi) d \xi$ since $u_{1}(0)=0$. Inserting in $\lambda u_{1}+\rho u_{1}^{\prime}-u_{2}^{\prime}=0$ yields

$$
-\left(1-\rho^{2}\right) u_{2}^{\prime}(\rho)+2 \lambda \rho u_{2}(\rho)+\lambda(\lambda-1) \int_{0}^{\rho} u_{2}(\xi) d \xi=0 .
$$

Set $u(\rho):=\int_{0}^{\rho} u_{2}(\xi) d \xi$. Then, $u \in H^{1}(0,1) \cap H_{\mathrm{loc}}^{2}(0,1), u(0)=0$ and $t_{0}(\lambda) u=0$. Thus, $u \in \operatorname{ker} T_{0}(\lambda)$.

Conversely, let $u \in \operatorname{ker} T_{0}(\lambda), u \neq 0$, and define $u_{1}(\rho):=\rho u^{\prime}(\rho)+(\lambda-1) u(\rho), u_{2}:=u^{\prime}$. Then, u $=\left(u_{1}, u_{2}\right)^{T} \in$ $\mathcal{H} \cap H_{\text {loc }}^{1}(0,1)^{2}, u_{1}(0)=0$ and $\ell_{0} \mathbf{u}=\lambda \mathbf{u}$. This shows $\mathbf{u} \in \operatorname{ker}\left(\lambda-L_{0}\right)$ and thus, $\lambda \in \sigma_{p}\left(L_{0}\right)$.

Suppose $\lambda-L_{0}$ is surjective and set $\mathbf{f}:=(f, 0)^{T} \in \mathcal{H}$. Then, there exists a $\mathbf{u} \in \mathcal{D}\left(L_{0}\right)$ such that $\left(\lambda-L_{0}\right) \mathbf{u}=\mathbf{f}$. As before, this implies that $u(\rho):=\int_{0}^{\rho} u_{2}(\xi) d \xi$ belongs to $\mathcal{D}\left(T_{0}(\lambda)\right)$ and satisfies $T_{0}(\lambda) u=f$. Thus, $T_{0}(\lambda)$ is surjective.

Conversely, if $T_{0}(\lambda)$ is surjective, we can find a $u \in \mathcal{D}\left(T_{0}(\lambda)\right)$ satisfying $T_{0}(\lambda) u(\rho)=f_{1}(\rho)+\rho f_{2}(\rho)+\lambda \int_{0}^{\rho} f_{2}(\xi) d \xi$ for any $\mathbf{f}=\left(f_{1}, f_{2}\right)^{T} \in \mathcal{H}$. Defining $\mathbf{u}$ by $u_{1}(\rho):=\rho u^{\prime}(\rho)+(\lambda-1) u(\rho)-\int_{0}^{\rho} f_{2}(\xi) d \xi$ and $u_{2}:=u^{\prime}$ we observe that $\mathbf{u} \in \mathcal{D}\left(L_{0}\right)$ and $\left(\lambda-L_{0}\right) \mathbf{u}=\mathbf{f}$ which shows surjectivity of $\lambda-L_{0}$.

Thus, we have shown that $\lambda-L_{0}$ is bijective if and only if $T_{0}(\lambda)$ is bijective. The closed graph theorem implies that $\left(\lambda-L_{0}\right)^{-1}$ is bounded if it exists. Finally, by basic ODE theory we conclude that $\operatorname{dim} \operatorname{ker} T_{0}(\lambda)$ is at most one-dimensional.

With Proposition 2 at hand we can easily calculate the spectrum of $L_{0}$ by solving a second order ODE. The complete characterization of $\sigma\left(L_{0}\right)$ is given in the following Lemma.

Lemma 4. The spectrum of $L_{0}$ is given by $\sigma\left(L_{0}\right)=\left\{\lambda \in \mathbb{C}: \operatorname{Re} \lambda \leq \frac{1}{2}\right\}$ where $\sigma_{p}\left(L_{0}\right)=\left\{\lambda \in \mathbb{C}: \operatorname{Re} \lambda<\frac{1}{2}\right\}$, $\sigma_{c}\left(L_{0}\right)=\left\{\lambda \in \mathbb{C}: \operatorname{Re} \lambda=\frac{1}{2}\right\}, \sigma_{r}\left(L_{0}\right)=\emptyset$.

Proof. First of all we note that the estimate $\left\|S_{0}(\tau)\right\| \leq e^{\frac{1}{2} \tau}$ for the semigroup $S_{0}$ generated by $L_{0}$ implies that $\lambda \in \rho\left(L_{0}\right)$ if $\operatorname{Re} \lambda>\frac{1}{2}$ (see e.g. Ref. 3, p. 55, Theorem 1.10 (ii)) and hence, we restrict ourselves to $\operatorname{Re} \lambda \leq \frac{1}{2}$.

The equation $t_{0}(\lambda) u=0$ can be solved explicitly and the solution $u_{0}(\cdot, \lambda)$, which satisfies $u_{0}(0, \lambda)=0$, is given by $u_{0}(\rho, \lambda)=(1-\rho)^{1-\lambda}-(1+\rho)^{1-\lambda}$. Thus, having Proposition 2 in mind we can immediately read off the point spectrum of $L_{0}$. We observe that $u_{0}(\cdot, \lambda) \in \mathcal{D}\left(T_{0}(\lambda)\right)$ if and only if $\operatorname{Re} \lambda<\frac{1}{2}$ which shows that any $\lambda$ with real part smaller than $\frac{1}{2}$ is an eigenvalue of $L_{0}$. Since the spectrum is always closed, we conclude that $\sigma\left(L_{0}\right)=\left\{\lambda \in \mathbb{C}: \operatorname{Re} \lambda \leq \frac{1}{2}\right\}$. Furthermore, by Ref. 3, p. 242, Proposition 1.10 we know that the topological boundary $\frac{1}{2}+i \mathbb{R}$ of $\sigma\left(L_{0}\right)$ is contained in the approximate point spectrum which is given by $\sigma_{p}\left(L_{0}\right) \cup \sigma_{c}\left(L_{0}\right)$ and we conclude that $\sigma_{c}\left(L_{0}\right)=\frac{1}{2}+i \mathbb{R}$. 
We have the interesting situation that $L_{0}$ possesses a continuum of eigenvalues. We remark that there exists a special subset $\{0,-1,-2, \ldots\}$ of eigenvalues with analytic eigenfunctions. However, from the point of view of semigroup theory there is no reason to consider those analytic eigenfunctions as distinguished. As a consequence we see that for any $\varepsilon>0$ there exist solutions of eq. (3) that grow like $\exp \left(\left(\frac{1}{2}-\varepsilon\right) \tau\right)$ for $\tau \rightarrow \infty$ which shows that the growth bound $\left\|S_{0}(\tau)\right\| \leq e^{\frac{1}{2} \tau}$ is sharp. This has implications for the original system in $(t, r)$ coordinates as well. Suppose one prescribes initial data determined by a nonanalytic mode solution. Although these modes are only defined for $r \leq T-t$, one can extend them in a $H^{1}$-fashion to yield perfectly admissible finite energy initial data. By causality the time development inside the past lightcone of $(t, r)=(T, 0)$ is independent of how the initial data have been extended and exactly determined by the behaviour of the mode solution. Hence, these modes are clearly seen to participate in generic time evolutions.

\section{An Estimate for $T_{0}^{-1}(\lambda)$}

The operator $L_{0}$ generates the strongly continuous semigroup $S_{0}$ which satisfies $\left\|S_{0}(\tau)\right\| \leq e^{\frac{1}{2} \tau}$ and this implies the resolvent estimate $\left\|R_{L_{0}}(\lambda)\right\| \leq \frac{1}{\operatorname{Re} \lambda-\frac{1}{2}}$ for all $\lambda \in \mathbb{C}$ with $\operatorname{Re} \lambda>\frac{1}{2}$ (cf. e.g. Ref. 3, p. 55, Theorem 1.10 (iii)) The resolvent $R_{L_{0}}(\lambda)$ can be given in terms of $T_{0}^{-1}(\lambda)$. To this end we define the operator $B(\lambda): \mathcal{H} \rightarrow L^{2}(0,1)$ by $B(\lambda) \mathbf{f}(\rho):=f_{1}(\rho)+\rho f_{2}(\rho)+\lambda \int_{0}^{\rho} f_{2}(\xi) d \xi$. A straightforward calculation shows that

$$
R_{L_{0}}(\lambda) \mathbf{f}(\rho)=\left(\begin{array}{c}
\rho\left(T_{0}^{-1}(\lambda) B(\lambda) \mathbf{f}\right)^{\prime}(\rho)+(\lambda-1) T_{0}^{-1}(\lambda) B(\lambda) \mathbf{f}(\rho)-\int_{0}^{\rho} f_{2}(\xi) d \xi \\
\left(T_{0}^{-1}(\lambda) B(\lambda) \mathbf{f}\right)^{\prime}(\rho)
\end{array}\right)
$$

The resolvent estimate implies $\left\|\left(T_{0}^{-1}(\lambda) B(\lambda) \mathbf{f}\right)^{\prime}\right\|_{L^{2}(0,1)} \leq \frac{1}{\operatorname{Re} \lambda-\frac{1}{2}}$ and the first component of $R_{L_{0}}(\lambda) \mathbf{f}$ then yields

$$
\left\|T_{0}^{-1}(\lambda) B(\lambda) \mathbf{f}\right\|_{L^{2}(0,1)} \leq \frac{1}{|\lambda-1|}\left(\frac{2}{\operatorname{Re} \lambda-\frac{1}{2}}+1\right)\|\mathbf{f}\|
$$

for all $\lambda$ with $\operatorname{Re} \lambda>\frac{1}{2}, \lambda \neq 1$. This estimate will be extremely useful later on.

\section{THE WAVE EQUATION WITH A POWER NONLINEARITY}

\section{A. Definition of the System, Known Results}

Consider the semilinear focusing wave equation

$$
\chi_{t t}-\Delta \chi-\chi^{p}=0
$$

for $\chi: \mathbb{R} \times \mathbb{R}^{3} \rightarrow \mathbb{R}$ where $p>1$ is an odd integer. This equation has been subject to many studies on nonlinear wave equations due to its simplicity. For the local well-posedness we refer to Lindblad and Sogge Ref. 7 and references therein.

Neglecting the Laplacian immediately leads to the homogeneous-in-space solution

$$
\chi_{0}(t, x)=c_{0}^{1 /(p-1)}(T-t)^{-2 /(p-1)}, c_{0}=\frac{2(p+1)}{(p-1)^{2}}
$$

which, together with smooth cut-off and finite speed of propagation, provides an example of a solution which starts from smooth compactly supported initial data and blows up for $t \rightarrow T-$. Based on numerics, it is conjectured that this solution describes the generic blow up scenario (cf. Ref. 2). For the system in one space dimension, this conjecture has been proved by Merle and Zaag Ref. 11. Furthermore, the blow up behaviour for certain ranges of $p$ and different space dimensions has been rigorously analysed in Ref. 8, Ref. 9, Ref. 10. In all of these studies the coordinate system $(\tau, \rho)$ plays an important role. We also mention the work of Galaktionov and Pohozaev Ref. 5 where a perturbative analysis in similarity coordinates is presented.

To analyse the stability of $\chi_{0}$ we linearize the problem by inserting the ansatz $\chi=\chi_{0}+\tilde{\psi}$ into Eq. (5) and neglecting higher order terms which leads to

$$
\tilde{\psi}_{t t}(t, x)-\Delta \tilde{\psi}(t, x)-p c_{0}(T-t)^{-2} \tilde{\psi}(t, x)=0 .
$$


We restrict ourselves to spherically symmetric perturbations and write $r=|x|$. As in Sec. 【A the substitution $\tilde{\psi} \mapsto \psi$ where $\psi(t, r)=r \tilde{\psi}(t, r)$ yields

$$
\psi_{t t}(t, r)-\psi_{r r}(t, r)-p c_{0}(T-t)^{-2} \psi(t, r)=0
$$

together with the regularity condition $\psi(t, 0)=0$ for all $t$. As before, we intend to study this equation in similarity coordinates $(\tau, \rho)$ and introduce the new dependent variables $\psi_{1}:=\psi_{t}$ and $\psi_{2}:=\psi_{r}$. Then, $\Psi:=\left(\psi_{1}, \psi_{2}\right)^{T}$ satisfies

$$
\partial_{t} \Psi(t, r)=\left(\begin{array}{ll}
0 & 1 \\
1 & 0
\end{array}\right) \partial_{r} \Psi(t, r)+p c_{0}(T-t)^{-2}\left(\begin{array}{ll}
0 & 1 \\
0 & 0
\end{array}\right) \int_{0}^{r} \Psi(t, s) d s
$$

with the regularity condition $\psi_{1}(t, 0)=0$ for all $t$. In similarity coordinates $(\tau, \rho)$ this system reads

$$
\partial_{\tau} \Phi(\tau, \rho)=\left(\begin{array}{cc}
-\rho & 1 \\
1 & -\rho
\end{array}\right) \partial_{\rho} \Phi(\tau, \rho)+p c_{0}\left(\begin{array}{ll}
0 & 1 \\
0 & 0
\end{array}\right) \int_{0}^{\rho} \Phi(\tau, \xi) d \xi
$$

where $\Phi(\tau, \rho):=\Psi\left(T-e^{-\tau}, \rho e^{-\tau}\right)$. Like in Sec. IIA the field $\phi(\tau, \rho):=\psi\left(T-e^{-\tau}, \rho e^{-\tau}\right)$ is determined by Eq. (2).

\section{B. Operator Formulation and Generation of Semigroup}

Let $\mathcal{H}:=L^{2}(0,1)^{2}$ and define $L^{\prime} \in \mathcal{B}(\mathcal{H})$ by

$$
L^{\prime} \mathbf{u}:=\left(\begin{array}{c}
p c_{0} \int_{0}^{\rho} u_{2}(\xi) d \xi \\
0
\end{array}\right) .
$$

It follows that

$$
\frac{d}{d \tau} \Phi(\tau)=\left(L_{0}+L^{\prime}\right) \Phi(\tau)
$$

for a function $\Phi:\left[\tau_{0}, \infty\right) \rightarrow \mathcal{H}$ is an operator formulation of Eq. (8) where $L_{0}$ is defined in Sec. [IB $\operatorname{and} \tau_{0}=-\log T$. Note also that $L^{\prime}$ is compact which will be useful later on.

Proposition 3. The operator $L:=L_{0}+L^{\prime}$ generates a strongly continuous one-parameter semigroup $S:[0, \infty) \rightarrow$ $\mathcal{B}(\mathcal{H})$ satisfying $\|S(\tau)\| \leq e^{\left(\frac{1}{2}+p c_{0}\right) \tau}$.

Proof. The claim follows immediately from Proposition 1 the Bounded Perturbation Theorem (see e.g. Ref. 3, p. 158, Theorem 1.3) and $\left\|L^{\prime}\right\| \leq p c_{0}$.

\section{Spectral Analysis of the Generator}

In order to improve the weak growth estimate from Proposition 3 we study the spectrum of $L$. Similar to the case of the free wave equation, the analysis of $\sigma(L)$ can be reduced to the study of an operator pencil defined on scalar functions. As a first step we calculate the point spectrum of $L$.

Lemma 5. $\lambda \in \sigma_{p}(L)$ if and only if $\operatorname{dim} \operatorname{ker}\left(T_{0}(\lambda)-p c_{0}\right)=1$.

The proof of this Lemma consists of an obvious modification of the first part of the proof of Proposition 2 and will therefore be omitted.

The equation $\left(t_{0}(\lambda)-p c_{0}\right) u=0$ has a regular singular point at $\rho=1$. Solutions of this equation can be given in terms of the hypergeometric function ${ }_{2} F_{1}$. Indeed, applying the substitution $\rho \mapsto z:=\rho^{2},\left(t_{0}(\lambda)-p c_{0}\right) u=0$ transforms into the hypergeometric differential equation

$$
z(1-z) v^{\prime \prime}(z)+[c-(a+b+1) z] v^{\prime}(z)-a b v(z)=0
$$

where $v(z):=u(\sqrt{z})$ and $a:=\frac{1}{4}\left(-1+2 \lambda-\sqrt{1+4 p c_{0}}\right), b:=\frac{1}{4}\left(-1+2 \lambda+\sqrt{1+4 p c_{0}}\right), c:=\frac{1}{2}$. Assume for the moment that $\lambda \neq 1$. Two linearly independent solutions $v_{1}, \tilde{v}_{1}$ are given by $v_{1}(z)={ }_{2} F_{1}(a, b ; a+b+1-c ; 1-z)$ and $\tilde{v}_{1}(z)=(1-z)^{c-a-b}{ }_{2} F_{1}(c-a, c-b ; c+1-a-b ; 1-z)$ (cf. Ref. 4). Hence, around $\rho=1$ there exist two linearly independent solutions $u_{1}$ and $\tilde{u}_{1}$ of $\left(t_{0}(\lambda)-p c_{0}\right) u=0$ where $u_{1}$ is analytic and $\tilde{u}_{1}$ has the form $\tilde{u}_{1}(\rho)=(1-\rho)^{1-\lambda} h(\rho)$ for an analytic function $h$ with $h(1) \neq 0$. If $\lambda=1$ then $c-a-b=0$ and we have a degenerate case. One solution is still given by $v_{1}$ as above and the second one diverges logarithmically for $z \rightarrow 1$. 
Lemma 6. The point spectrum of $L$ is given by $\sigma_{p}(L)=\sigma_{p}\left(L_{0}\right) \cup\left\{1+\frac{2}{p-1}\right\}$. Moreover, we have $\sigma(L) \supset \sigma\left(L_{0}\right)$.

Proof. The asymptotic behaviour of solutions of $\left(t_{0}(\lambda)-p c_{0}\right) u=0$ for $\rho \rightarrow 1$ is the same as for the analogous problem for the free wave equation (Sec. IIC) and thus, for $\operatorname{Re} \lambda<\frac{1}{2}$ both linearly independent solutions are $H^{1}$ near $\rho=1$. This shows $\left\{\lambda \in \mathbb{C}: \operatorname{Re} \lambda<\frac{1}{2}\right\} \subset \sigma_{p}(L)$. Thus, we restrict ourselves to $\operatorname{Re} \lambda>\frac{1}{2}$. Consider the following solutions $v_{1}(z):={ }_{2} F_{1}(a, b ; a+b+1-c ; 1-z), v_{0}(z):=z^{1-c}{ }_{2} F_{1}(a+1-c, b+1-c ; 2-c ; z)$ and $\tilde{v}_{0}(z):={ }_{2} F_{1}(a, b ; c ; z)$ of Eq. (91). Since $v_{0}$ and $\tilde{v}_{0}$ are linearly independent, there exist constants $c_{1}, c_{2}$ such that $v_{1}=c_{1} \tilde{v}_{0}+c_{2} v_{0}$. The solution $v_{1}$, which is analytic around $\rho=1$, satisfies the boundary condition $v_{1}(0)=0$ if and only if $c_{1}=0$ and exactly in this case, $\operatorname{ker}\left(T_{0}(\lambda)-p c_{0}\right) \neq\{0\}$ since the other linearly independent solution around $\rho=1$ does not belong to $H^{1}$. The coefficient $c_{1}$ can be given in terms of the $\Gamma$-function (cf. Ref. 4) and reads

$$
c_{1}=\frac{\Gamma(a+b+1-c) \Gamma(1-c)}{\Gamma(a+1-c) \Gamma(b+1-c)} .
$$

Since the Gamma function does not have zeros, $c_{1}=0$ if and only if $a+1-c$ or $b+1-c$ is a pole which yields the condition $a+1-c=-k$ or $b+1-c=-k$ for $k=0,1, \ldots$ There is only one solution with $\operatorname{Re} \lambda>\frac{1}{2}$ and it is given by $\lambda=1+\frac{2}{p-1}$.

Since the spectrum is closed, the line $\frac{1}{2}+i \mathbb{R}$ belongs to $\sigma(L)$ and we conclude $\sigma(L) \supset \sigma\left(L_{0}\right)$.

As a consequence of the compactness of $L^{\prime}$ we are able to determine the whole spectrum of $L$ by considering solutions of the ODE $\left(T_{0}(\lambda)-p c_{0}\right) u=0$. This will be shown in the next Lemma.

Lemma 7. We have $\lambda \in \rho(L)$ if and only if $\lambda \in \rho\left(L_{0}\right)$ and $I-L^{\prime} R_{L_{0}}(\lambda)$ is bounded invertible. Furthermore, $\sigma(L)=\sigma\left(L_{0}\right) \cup\left\{1+\frac{2}{p-1}\right\}$.

Proof. Let $\lambda \in \rho(L)$. Then, by Lemma $6, \lambda \in \rho\left(L_{0}\right)$ and the identity

$$
\lambda-L=\left(I-L^{\prime} R_{L_{0}}(\lambda)\right)\left(\lambda-L_{0}\right)
$$

shows that $\left(I-L^{\prime} R_{L_{0}}(\lambda)\right)^{-1}$ exists and belongs to $\mathcal{B}(\mathcal{H})$. Conversely, suppose $\lambda \in \rho\left(L_{0}\right)$ and $\left(I-L^{\prime} R_{L_{0}}(\lambda)\right)^{-1} \in \mathcal{B}(\mathcal{H})$. Then, by using Eq. (10) again we conclude that $\lambda \in \rho(L)$.

Now let $\lambda \in \sigma(L) \backslash \sigma\left(L_{0}\right)$. By the first part this implies $1 \in \sigma\left(L^{\prime} R_{L_{0}}(\lambda)\right)$. From the compactness of $L^{\prime} R_{L_{0}}(\lambda)$ we conclude that $1 \in \sigma_{p}\left(L^{\prime} R_{L_{0}}(\lambda)\right)$ which means that there exists a $\mathbf{f} \in \mathcal{H}, \mathbf{f} \neq 0$, with $\left(I-L^{\prime} R_{L_{0}}(\lambda)\right) \mathbf{f}=0$. Set $\mathbf{u}:=\left(\lambda-L_{0}\right)^{-1} \mathbf{f}$. Then, $\mathbf{u} \neq 0, \mathbf{u} \in \mathcal{D}\left(L_{0}\right)=\mathcal{D}(L)$ and Eq. (10) shows that $(\lambda-L) \mathbf{u}=0$ which implies $\lambda \in \sigma_{p}(L)$. Hence, any spectral value of $L$ which does not belong to $\sigma\left(L_{0}\right)$ is an eigenvalue and by Lemma $6,1+\frac{2}{p-1}$ is the only eigenvalue of $L$ outside $\sigma\left(L_{0}\right)$.

We give an explanation how the existence of the single isolated (unstable) eigenvalue $\lambda_{0}:=1+\frac{2}{p-1}$ can be understood. Consider a nonlinear equation $F(u)=0$ where $F: U \subset X \rightarrow Y$ is a Fréchet differentiable nonlinear mapping from an open subset $U$ of a Banach space $X$ to a Banach space $Y$. Suppose there exists a one-parameter family $\left\{u_{s} \in U: s \in(a, b)\right\}$ of solutions (i.e. $F\left(u_{s}\right)=0$ for all $s \in(a, b)$ ) such that the mapping $s \mapsto u_{s}$ : $(a, b) \rightarrow X$ is (strongly) differentiable at $s_{0} \in(a, b)$. An application of the chain rule shows that $0=\left.\frac{d}{d s}\right|_{s=s_{0}} F\left(u_{s}\right)=$ $\left.D F\left(u_{s_{0}}\right) \frac{d}{d s}\right|_{s=s_{0}} u_{s}$ and therefore, $\left.\frac{d}{d s}\right|_{s=s_{0}} u_{s} \in X$ is a solution of the linearized problem $D F\left(u_{s_{0}}\right) u=0$ where $D F$ is the Fréchet derivative of $F$.

Note that we can choose the blow up time $T$ freely, hence, the solution $\chi_{0}(t, r)=c_{0}^{1 /(p-1)}(T-t)^{-2 /(p-1)}$ is a one-parameter family of solutions rather than a single one. A direct calculation shows that $\psi(t, r):=r \frac{d}{d T} \chi_{0}(t, r)$ solves the linearized problem eq. (7) and in similarity coordinates, $\psi_{r}$ is given by $C e^{\lambda_{0} \tau}$. Therefore, the existence of the unstable eigenvalue $\lambda_{0}$ is a direct consequence of the time translation symmetry of the original problem. Actually, we are only interested in "stability modulo this symmetry" and in the next section we will show how to make this idea rigorous.

\section{Spectral Projection on the Stable Subspace}

We want to restrict the set of admissible perturbations in such a way that the instability caused by the time translation symmetry does not contribute to the linear time evolution. The spectrum of $L$ is separated in a bounded part (which consists of the single eigenvalue $1+\frac{2}{p-1}$ ) and an unbounded part $\sigma\left(L_{0}\right)$. Let $\Gamma$ be a circle in the complex 
plane with center $1+\frac{2}{p-1}$ and radius smaller than 1 , i.e. $\Gamma \subset \rho(L)$. Then we can define the spectral projection $P \in \mathcal{B}(\mathcal{H})$ by

$$
P:=\frac{1}{2 \pi i} \int_{\Gamma} R_{L}(\lambda) d \lambda
$$

which projects onto the closed subspace $\mathcal{M}:=P \mathcal{H} \subset \mathcal{H}$. The operator $P$ commutes with $L$ and we have the decomposition $\mathcal{H}=\mathcal{M} \oplus \mathcal{N}$ where $\mathcal{N}:=(I-P) \mathcal{H}$. Furthermore, $L$ is decomposed into two parts $L_{\mathcal{M}}:=\left.L\right|_{\mathcal{D}(L) \cap \mathcal{M}}$ and $L_{\mathcal{N}}:=\left.L\right|_{\mathcal{D}(L) \cap \mathcal{N}}$ acting on $\mathcal{M}$ and $\mathcal{N}$, respectively, and $\sigma\left(L_{\mathcal{M}}\right)=\left\{1+\frac{2}{p-1}\right\}$ whereas $\sigma\left(L_{\mathcal{N}}\right)=\sigma\left(L_{0}\right)($ see Ref. 6 , p. 178, Theorem 6.17). However, we remark that the eigenvalues with analytic eigenfunctions are not the same for $L_{0}$ and $L_{\mathcal{N}} . L_{\mathcal{N}}$ inherits all the nice properties of $L$, i.e. $L_{\mathcal{N}}$ is densely defined, closed and the resolvent of $L_{\mathcal{N}}$ is given by $\left.R_{L}(\lambda)\right|_{\mathcal{N}}$. Thus, $L_{\mathcal{N}}$ generates a strongly continuous semigroup $S_{\mathcal{N}}$ on $\mathcal{N}$ satsfying $\left\|S_{\mathcal{N}}(\tau)\right\| \leq e^{\left(\frac{1}{2}+p c_{0}\right) \tau}$. The semigroup $S_{\mathcal{N}}$ describes the linear time evolution "modulo the instability caused by the time translation symmetry of the original problem". However, since the generator $L_{\mathcal{N}}$ is not normal, we cannot directly obtain a sharp growth bound for $S_{\mathcal{N}}$ from the spectral properties of $L_{\mathcal{N}}$.

The interesting notion concerning the qualitative behaviour of $S_{\mathcal{N}}$ is the growth bound $\omega_{0}\left(S_{\mathcal{N}}\right)$ which is defined by

$$
\omega_{0}\left(S_{\mathcal{N}}\right):=\inf \left\{s \in \mathbb{R}: \exists C_{s} \geq 1:\left\|S_{\mathcal{N}}(\tau)\right\| \leq C_{s} e^{s \tau} \text { for all } \tau>0\right\}
$$

It is well-known (cf. e.g. Ref. 3) that

$$
\omega_{0}\left(S_{\mathcal{N}}\right)=\inf \left\{\kappa>s\left(L_{\mathcal{N}}\right): \sup _{\omega \in \mathbb{R}}\left\|\left.R_{L}(\kappa+i \omega)\right|_{\mathcal{N}}\right\|<\infty\right\}
$$

where $s\left(L_{\mathcal{N}}\right):=\sup \left\{\operatorname{Re} \lambda: \lambda \in \sigma\left(L_{\mathcal{N}}\right)\right\}$ is the spectral bound of $L_{\mathcal{N}}$. In our case we have $s\left(L_{\mathcal{N}}\right)=\frac{1}{2}$ and thus, we have to study the behaviour of $R_{L}(\lambda)$ for $\operatorname{Re} \lambda>\frac{1}{2}$ and $|\operatorname{Im} \lambda| \rightarrow \infty$.

Lemma 8. There exists a $C>0$ such that $\left\|R_{L}(\lambda)\right\| \leq C$ for all $\lambda \in \rho(L)$.

Proof. Let $\operatorname{Re} \lambda>\frac{1}{2}$ and $\lambda \neq 1+\frac{2}{p-1}$. Applying Lemma 7 and the identity Eq. (10) we obtain $R_{L}(\lambda)=R_{L_{0}}(\lambda)(I-$ $\left.L^{\prime} R_{L_{0}}(\lambda)\right)^{-1}$. The operator $L^{\prime} R_{L_{0}}(\lambda)$ is given by

$$
L^{\prime} R_{L_{0}}(\lambda) \mathbf{f}=\left(\begin{array}{c}
p c_{0} T_{0}^{-1}(\lambda) B(\lambda) \mathbf{f} \\
0
\end{array}\right)
$$

Thus, the estimate Eq. (4) implies

$$
\left\|L^{\prime} R_{L_{0}}(\lambda)\right\| \leq \frac{p c_{0}}{|\lambda-1|}\left(\frac{2}{\operatorname{Re} \lambda-\frac{1}{2}}+1\right)
$$

and this shows that $\left\|L^{\prime} R_{L_{0}}(\lambda)\right\| \rightarrow 0$ for $|\operatorname{Im} \lambda| \rightarrow \infty$. We conclude that $I-L^{\prime} R_{L_{0}}(\lambda) \rightarrow I$ and hence, $(I-$ $\left.L^{\prime} R_{L_{0}}(\lambda)\right)^{-1} \rightarrow I$ for $|\operatorname{Im} \lambda| \rightarrow \infty$ uniformly. Therefore, the claim follows from the uniform boundedness of $\left\|R_{L_{0}}(\lambda)\right\|$ for $\operatorname{Re} \lambda>\frac{1}{2}$.

Lemma 8 shows that $\omega_{0}\left(S_{\mathcal{N}}\right)=\frac{1}{2}$ and thus, the growth bound of the semigroup $S_{\mathcal{N}}$ coincides with the spectral bound of its generator. We immediately conclude the linear asymptotic stability of the solution $\chi_{0}$ with respect to the $L^{\infty}$ norm (cf. Sec. [A ).

\section{ACKNOWLEDGMENTS}

The author wants to thank Peter C. Aichelburg and Piotr Bizoń for helpful discussions. This work has been supported by the Austrian Fonds zur Förderung der wissenschaftlichen Forschung (FWF) Project No. P19126.

[1] Horst Reinhard Beyer. Beyond partial differential equations, volume 1898 of Lecture Notes in Mathematics. Springer, Berlin, 2007. On linear and quasi-linear abstract hyperbolic evolution equations. 
[2] Piotr Bizoń, Tadeusz Chmaj, and Zbisław Tabor. On blowup for semilinear wave equations with a focusing nonlinearity. Nonlinearity, 17(6):2187-2201, 2004.

[3] Klaus-Jochen Engel and Rainer Nagel. One-parameter semigroups for linear evolution equations, volume 194 of Graduate Texts in Mathematics. Springer-Verlag, New York, 2000. With contributions by S. Brendle, M. Campiti, T. Hahn, G. Metafune, G. Nickel, D. Pallara, C. Perazzoli, A. Rhandi, S. Romanelli and R. Schnaubelt.

[4] Arthur Erdélyi, Wilhelm Magnus, Fritz Oberhettinger, and Francesco G. Tricomi. Higher transcendental functions. Vol. I. Robert E. Krieger Publishing Co. Inc., Melbourne, Fla., 1981. Based on notes left by Harry Bateman, With a preface by Mina Rees, With a foreword by E. C. Watson, Reprint of the 1953 original.

[5] V. A. Galaktionov and S. I. Pohozaev. On similarity solutions and blow-up spectra for a semilinear wave equation. Quart. Appl. Math., 61(3):583-600, 2003.

[6] Tosio Kato. Perturbation theory for linear operators. Classics in Mathematics. Springer-Verlag, Berlin, 1995. Reprint of the 1980 edition.

[7] Hans Lindblad and Christopher D. Sogge. On existence and scattering with minimal regularity for semilinear wave equations. J. Funct. Anal., 130(2):357-426, 1995.

[8] Frank Merle and Hatem Zaag. Determination of the blow-up rate for the semilinear wave equation. Amer. J. Math., 125(5):1147-1164, 2003.

[9] Frank Merle and Hatem Zaag. Determination of the blow-up rate for a critical semilinear wave equation. Math. Ann., 331(2):395-416, 2005.

[10] Frank Merle and Hatem Zaag. On growth rate near the blowup surface for semilinear wave equations. Int. Math. Res. Not., (19):1127-1155, 2005.

[11] Frank Merle and Hatem Zaag. Existence and universality of the blow-up profile for the semilinear wave equation in one space dimension. J. Funct. Anal., 253(1):43-121, 2007.

[12] Roland Donninger. Asymptotics and analytic modes for the wave equation in similarity coordinates. J. Evol. Equ., 9(3):511-523, 2009.

[13] Roland Donninger. Nonlinear stability of self-similar solutions for semilinear wave equations. Preprint arXiv:0811.1908, to appear in Comm. Partial Differential Equations., 2008. 